\title{
RECURRENCE OF SYMBOLIC ELEMENTS IN DYNAMICS ${ }^{1}$
}

\author{
RUFUS OLDENBURGER
}

1. Introduction. Morse and Hedlund [1] have given a symbolic treatment of modern theoretical dynamics as developed by Birkhoff [2] and others. In the Morse-Hedlund viewpoint the concept of recurrence plays an important role. To establish various theorems on symbolic trajectories Morse and Hedlund introduced "symbolic elements," the analogues of line elements on ordinary trajectories, a symbolic element being the notion of a trajectory $T$ and a particular symbol in $T$. In the present paper we shall be concerned primarily with the question: "How are recurrence of a trajectory $T$ and elements based on T related?"

2. Definitions. For terms defined elsewhere and used here the reader is referred to other papers $[1,3]$. Let rays $R_{i}(i=1,2,3,4)$ be given by

$$
\alpha_{i 1} \alpha_{i 2} \alpha_{i 3} \cdots \text {. }
$$

The distance $E_{1} E_{2}$ between the elements $E_{1}=\left(R_{1}, R_{2}\right)$ and $E_{2}=\left(R_{3}, R_{4}\right)$ is defined to be $1 / n$ where $n$ is such that $a_{1 j}=a_{3 j}, a_{2 j}=a_{4 j}$ for each value of $j$ in the range $1,2, \cdots, n$, while

$$
\left(a_{1, n+1}, a_{2, n+1}\right) \neq\left(a_{3, n+1}, a_{4, n+1}\right) .
$$

The element $E_{1}$ is the ray

$$
A_{1} A_{2} A_{3} \cdots,
$$

where $A_{j}$ denotes the pair of symbols $\left(a_{1 j}, a_{2 j}\right)$. In what follows the term "recurrence of $E_{1}$ " designates the recurrence of the ray (1). We recall that a ray (1) is recurrent if for each $n$ there is an $m$ such that each $n$-block in (1) is contained in each $m$-block of (1). For each $n$ the value $R(n)$ of the recurrency function of (1) is the smallest $m$ with the property just mentioned.

3. Recurrence. It is evident that the recurrence of a single element based on a trajectory $T$ does not imply the recurrence of $T$.

THEOREM 1. If each element based on a trajectory $T$ is recurrent, $T$ is recurrent.

The recurrence of each ray based on $T$ obviously implies the recur-

\footnotetext{
${ }_{1}^{1}$ Presented to the Society, December 28, 1939, under the title Symbolic elements in dynamics.
} 
rence of $T$. The recurrence of each element based on $T$ implies the recurrence of each ray based on $T$, whence $T$ is recurrent.

THEOREM 2. There exists a recurrent trajectory with a non-recurrent element.

If $\alpha$ is irrational, the trajectory $T(0, \alpha)$ of Morse and Hedlund [4] is recurrent and is of the form $R^{-1} a b R$. The element $(b R, a R)$ is not recurrent.

The proof of the theorem to follow is simple and will be omitted.

Theorem 3. A trajectory $T$ is identical with its inverse if and only if $T=R^{-1} R$ or $T=R^{-1} a R$ for some ray $R$ and symbol $a$.

Theorem 4. If a trajectory $T$ is recurrent and is identical with its inverse, each element based on $T$ is recurrent.

We assume first that $T=R^{-1} R$ for some ray $R$. Consider an element $E=\left(B^{-1} R, R_{1}\right)$, where $B$ is such that $R=B R_{1}$. We permit $B$ to be vacuous, in which case $E=\left(R, R_{1}\right)$. Let $G$ denote the leading $s$-block of $R_{1}$ for a given value of $s$, and let $r$ denote the length of $B$. Let $J$ denote the leading $s$-block of $B^{-1} R$. Then $J$ in $B^{-1} R$ matches $G$ in $R_{1}$, and the pair of blocks $J, G$ corresponds to the block $A_{1} \cdots A_{s}$ in the representation (1) of $E$. The block $G^{-1} B^{-1} B G$ is a subblock of $T$. Let $R(n)$ denote the recurrency function of $T$, whence $G^{-1} B^{-1} B G$ occurs in each $R(2 r+2 s)$-block of $T$. Let $n$ be the number of symbols preceding one such block $G^{-1} B^{-1} B G$ in $R$, so that

$$
R=H G^{-1} B^{-1} B G R_{2},
$$

where $H$ is a block of length $n$ and $R_{2}$ is a subray of $R$. For brevity we introduce the notation $K=G^{-1} B^{-1} B G$ so that $R=H K R_{2}$. It is no restriction on the generality of the argument to follow to suppose that $n \geqq r$ so that the block $K$ is a subblock of $R_{1}$. The final block $G$ in $K$ is preceded in $R_{1}$ by a subblock of length $n+r+s$. In the ray $B^{-1} R$ the final subblock $B^{-1} B G$ of $K$ is preceded by a block of length $n+r+s$. Thus the initial $s$-block $J$ of the subblock $B^{-1} B G$ of $K$ in $B^{-1} R$ matches the final block $G$ of $K$ in $R_{1}$. We have proved that in each $R(2 r+2 s)$-block of $R_{1}$ there is a subblock $G$ matching a subblock $J$ of $B^{-1} R$ whence each $R(2 r+2 s)$-block in the representation (1) contains a block identical with $A_{1} \cdots A_{s}$. Since each block in (1) is contained in the block $A_{1} \cdots A_{s}$ for large enough $s$, it follows that $E$ is recurrent.

We suppose, finally, that $T=R^{-1} a R$ for some ray $R$ and symbol $a$. Let $r$ and $s$ be arbitrary non-negative integers. We consider an ele- 
ment $E=\left(D^{-1} a R, R_{1}\right)$ where $D$ is the leading $r$-block of $R$ so that $R=D R_{1}, R_{1}$ being a subray of $R$. We let $G$ denote the leading $s$-block of $R_{1}$ so that $R_{1}=G R_{2}$ where $R_{2}$ is a subray of $R_{1}$. Evidently, $T$ contains the subblock $G^{-1} D^{-1} a D G$ and the initial block $G$ of $R_{1}$ matches the leading $s$-block $J$ of $D^{-1} a R$. The last block $G$ in any block $G^{-1} D^{-1} a D G$ of $R_{1}$ matches the leading block $J$ of a subblock $D^{-1} a D G$ in $D^{-1} a R$, whence $E$ is recurrent.

By Theorem 3 the above argument proves that each element $E$ based on $T$ is recurrent.

THEOREM 5. If for some block $C$ and ray $R$ a trajectory $T$ is of the form $R^{-1} C R$, and if each element based on $T$ is recurrent, the trajectory $T$ is identical with its inverse.

We suppose first that $C$ is of even length $2 q$, and we accordingly write $C=C_{1} C_{2}$ where $C_{1}$ and $C_{2}$ are of length $q$. We consider the element $E=\left(C_{1}^{-1} R, C_{2} R\right)$ based on $T$. Since $E$ is recurrent, $C_{2}$ occurs in the subray $R$ matched in $C_{2} R$ by a block $C_{1}^{-1}$ in $C_{1}^{-1} R$. It follows that $C_{1}^{-1}$ matches $C_{2}$ in $R$, whence $C_{1}=C_{2}^{-1}$ and $T=T^{-1}$.

If now $C$ is of odd length $2 q+1$, we write $C=C_{1} a C_{3}$ where $C_{1}$ and $C_{3}$ are of length $q$. Let the initial symbol of $R$ be denoted by $b$. Since the element $E=\left(C_{1}^{-1} R, a C_{3} R\right)$ is recurrent, there is a subblock $a C_{3}$ of $R$ matched in $a C_{3} R$ by a block $C_{1}^{-1} b$ in the ray $C_{1}^{-1} R$. Hence there is a block $C_{3}$ in $R$ matching a block $C_{1}^{-1}$ in $R$. Thus $C_{1}=C_{3}^{-1}$ and $T=T^{-1}$.

The Morse recurrent trajectory $T$ extensively studied in the literature is a trajectory identical with its inverse, whence each element based on $T$ is recurrent. Morse and Hedlund gave methods termed projection, reduction, association, and substitution by which one can obtain a recurrent trajectory from a given recurrent trajectory. One can prove readily that the elements based on the trajectories obtained by derivation from the Morse recurrent trajectory are recurrent.

Elsewhere the author proved (3) that the exponent trajectory $T_{e}$ of a recurrent trajectory $T$ is generated by a finite number of symbols, and is recurrent. Let $T_{1}, T_{2}, \cdots$ be a sequence of trajectories such that $T_{i}$ is the exponent trajectory of $T_{i-1}$ for each $i$. We term $T_{2}, T_{3}, \cdots$ the successive exponent trajectories of $T$. One can prove readily that the successive exponent trajectories of the Morse recurrent trajectory are each of the form $R^{-1} a R$ where $a$ is a symbol and $R$ is a ray, whence the elements based on these successive trajectories are recurrent.

We let $R_{i}(n)$ denote the recurrency function of the element $E_{i}$ based on a trajectory $T$. If for each $i$ the set $R_{i}(1), R_{i}(2), \cdots$ is bounded, $T$ is said to be E-recurrent. For the Morse recurrent trajectory one 
can prove that the set $R_{i}(1)$ is not bounded whence this trajectory is not $E$-recurrent. The existence of a nonperiodic $E$-recurrent trajectory is still an open question.

\section{REFERENCES}

1. M. Morse and G. A. Hedlund, Symbolic dynamics, American Journal of Mathematics, vol. 60 (1938), pp. 815-866.

2. G. D. Birkhoff, Dynamical Systems, American Mathematical Society Colloquium Publications, vol. 9, New York, 1927.

3. R. Oldenburger, Exponent trajectories in symbolic dynamics, Transactions of this Society, vol. 46 (1939), pp. 453-466.

4. Morse and Hedlund, Symbolic dynamics, II. Sturmian trajectories, American Journal of Mathematics, vol. 52 (1940), pp. 1-42.

ILLinois Institute of TeChNOLOGY 